\title{
Classifying snakebite in South Africa: Validating a scoring system
}

\author{
D Wood, ${ }^{1}$ MB BCh, FCEM, MPhil; B Sartorius, ${ }^{2} \mathrm{PhD}$; $\mathbf{R}$ Hift, ${ }^{1} \mathrm{MB}$ BCh, FRCP, PhD \\ ${ }^{1}$ School of Clinical Medicine, College of Health Sciences, University of KwaZulu-Natal, Durban, South Africa \\ ${ }^{2}$ School of Public Health, College of Health Sciences, University of KwaZulu-Natal, Durban, South Africa
}

Corresponding author: D Wood (darrylrwood@yahoo.co.uk)

Objective. To develop and validate a scoring system for managing snakebites in South Africa (SA).

Methods. We studied all snakebite admissions to a regional hospital in KwaZulu-Natal, SA. The primary outcome was an active treatment intervention (ATI) defined as antivenom treatment or any surgical procedure. The development cohort consisted of 879 patients with snakebite who presented to the Ngwelezane Hospital Emergency Department from December 2008 to December 2013. Factors predictive of ATI and the optimal cut-off score for predicting an ATI were identified. These factors were then used to develop a standard scoring system. The score was then tested prospectively for accuracy in a new validation cohort consisting of 100 patients admitted for snakebite to our unit from 1 December 2014 to 31 March 2015. Accuracy of the score was determined.

Results. Of 879 snakebite admissions, 146 in the development cohort and 40 of 100 in the development validation cohort reached the primary endpoint of an ATI. Six risk predictors for ATI were identified from the development cohort: age <14 years (odds ratio (OR) 2.13), delay to admission $>7$ hours (OR 4.63), white cell count $>10 \times 10^{9} / \mathrm{L}$ (OR 3.15), platelets $<92 \times 10^{9} / \mathrm{L}$ (OR 2.35), haemoglobin $<7.1 \mathrm{~g} / \mathrm{dL}$ (OR 5.68), international normalised ratio $>1.2$ (OR 2.25). Each risk predictor was assigned a score of 1; receiver operating characteristic curve analysis returned a value of $>4$ out of 6 as the optimal cut-off for prediction of an ATI (area under the curve 0.804 ; $95 \%$ confidence interval $0.758-0.84$ ). Testing of the score on the validation cohort produced a specificity of $96.6 \%$ and a sensitivity of $22.5 \%$. The positive predictive value and negative predictive value were $81.8 \%$ and $65.2 \%$, respectively.

Conclusion. Our results show that the identified score is a useful adjunct to clinical assessment in managing snakebite. Its value is greatest when used in those patients who fall in the mild to moderate clinical category. Until our severity score has been validated (or modified) for use across SA, we propose to name it the Zululand Severity Score; a true SA Severity Score may follow.

S Afr Med J 2017;107(1):46-51 DOI:10.7196/SAMJ.2017.v107i1.11361

There are some 38 venomous snake species in South Africa (SA), of which approximately half are dangerous to humans. ${ }^{[1]}$ Snake species and density vary across the regions of SA. ${ }^{[2]}$ Prevalent species include the Mozambique spitting cobra (Naja mossambica) and puff adder (Bitis arietans), an elapid and viperid respectively, both of which have a potent cytotoxic venom. The black mamba (Dendroaspis polylepis) and various cobra (Naja) species are elapids possessing potent neurotoxic venom and muscle weakness. Rarely, the boomslang (Dispholidus typus), a colubrid with a haemorrhagic venom, can cause potentially fatal bleeding.

The severity of a snakebite is highly variable and dependent on numerous factors. Snakes will utilise their venom differently depending on the situation, controlling the volume injected and the fang contact time with their prey ${ }^{[3]}$ Defence bites that are designed to fend off danger may in some cases deliver less or no venom, resulting in a dry bite. ${ }^{[3-6]}$ The potency of venom varies with the species of snake and in larger snakes the volume of expelled venom is usually higher ${ }^{[3,5]}$ In the case of cytotoxic bites, the severity of injury is critically dependent on the body part bitten and the depth at which the venom is injected.

In the absence of objective criteria, the presumed severity of a bite is typically dependent on the attending doctor's own experience and clinical judgement. This process of establishing severity is highly subjective and is included in guidelines for snakebite management in SA. ${ }^{[7]}$ The severity of envenomation may not be initially appreciated by admitting doctors. Cytotoxic swelling may progress insidiously, as may the effects of systemic toxicity, resulting in shock. In addition, coagulopathy may not be identified until the patient manifests with lethal haemorrhaging.

Guidelines have been developed to assist doctors to treat patients with snakebite. The recommendations on which our own institution's treatment protocol is based were developed in 2005 and are largely derived from the knowledge and experience of a single surgeon. ${ }^{[7]}$

Most guidelines are based on clinical grading systems that rely on the clinician's ability to evaluate the clinical manifestations evident in the patient. A number of scoring systems for use in North American crotalid bites have been published. ${ }^{[8-11]}$ Coagulopathy is a major feature of such bites and these systems typically include coagulation studies. Such systems are unlikely to be directly transferable to the bites of other snakes with a different toxicity profile. Dart et al. ${ }^{[12]}$ proposed a complex guide for crotalid bites in the form of a detailed scoring system, the Snakebite Severity Score (SSS). This detailed scoring system is limited by a reliance on subjective judgement by the physician, the time it takes to do the score in the clinical setting and the fact that it was validated retrospectively. Scharman and Noffsinger ${ }^{[13]}$ modified the SSS score for copperhead snakebites by excluding the coagulation abnormality parameter and adding a progression of swelling parameter to produce a score based on clinical assessment alone. Gold et al ${ }^{[5]}$ described three parameters to guide clinicians when assessing snakebite in the USA. Two of these parameters are clinical; the third parameter is the presence of coagulation abnormalities. Some authorities find the published scoring systems to have little value in the clinical setting and believe they are of more value in the research setting than in clinical practice. ${ }^{[14]}$ 
That said, the need for an objective and reliable scoring system persists. Bites from the most venomous SA snakes are often severe and may be fatal; there is therefore an imperative to identify the more severe cases early and to treat them vigorously. On the other hand, the mainstay of treatment in $\mathrm{SA}$ is a polyvalent equine antiserum with a venerable ancestry extending back to 1928. Initially a bivalent antiserum, it is now raised against venom derived from 10 species. However, its use is associated with a high rate of anaphylaxis and it is essential that its use is restricted to patients who really require it. ${ }^{[15]}$

We therefore undertook a study to identify the factors most strongly predictive of an adverse outcome in our population, arising from which we propose a scoring system called the Zululand Snakebite Score (ZSS) that is validated in our setting. We suggest that its greatest value is in identifying those patients who do not appear overtly seriously affected at first presentation, but are at high risk of progression to severity over the ensuing hours. In such cases intensive monitoring and repeated evaluation are mandatory, and the early use of antivenom may be justified.

\section{Methods \\ Setting and participants}

The Ngwelezane Hospital serves a population of 3 million and is the referral hospital for 20 district hospitals in the subtropical northeast of SA. This region has a very high incidence of snakebite. ${ }^{[15-19]}$ The Emergency Department sees approximately 60000 new patients annually. In the summer months, up to $10 \%$ of admissions are due to snakebite. All snakebite admissions to the Emergency Department from the local Uthungulu district and referrals from further regions such as the districts of Zululand and Umkhanyekhude were included in the study. The patient history of a bite and the evidence of fang marks confirmed the diagnosis of snakebite. Presentations ranged from mild (often dry bites) to severe limb- or life-threatening envenomation. All patients were reviewed by an emergency medicine specialist and treated with a standard snakebite protocol based on the recommendations of Blaylock. ${ }^{[7]}$ All patients had baseline blood tests taken on admission and received basic treatment that included analgesia, fluids and tetanus toxoid.

\section{Outcomes}

The primary outcome was an active treatment intervention (ATI), which we defined as the administration of antivenom or a surgical procedure at any stage during admission. The decision to treat a patient with antivenom was made by senior emergency medicine specialists with extensive experience in snakebite. The indications for antivenom treatment were: severe cytotoxic swelling, active bleeding in the case of haemotoxic envenomation, or symptomatic weakness in neurotoxic envenomations. The surgical procedures were defined as debridement of wound necrosis, skin grafts, fasciotomy for compartment syndrome or amputations, and the decision for this was made by a senior surgeon with experience in the management of snakebite.

\section{Study design}

We performed a validation study using two separate patient cohorts (Fig. 1). The development cohort consisted of all patients with snakebite who presented to the Ngwelezane Hospital Emergency Department from December 2008 to December 2013. Data from 983 admissions over this time were retrieved and analysed retrospectively. Factors predictive of ATI were identified using bivariate and multivariable adjusted logistic regression. Receiver operating characteristic (ROC) curve analysis was performed and the optimal cut-off score for predicting an ATI identified. These factors were then used to develop a standard scoring system.

The score was then tested prospectively for accuracy in a new validation cohort consisting of all patients admitted for snakebite during the high-incidence season under the same criteria from 1 December 2014 to 31 March 2015. In addition, the optimal cut-off score in the validation cohort was also determined and compared with the optimal cut-off score in the development cohort. All other factors were unchanged between the two cohorts, including the definitions of ATI, the indications for surgery or antivenom, treatment protocols and the identities of the senior staff supervising therapy.

\section{Statistical analysis}

Data were stored on a protected Excel database, version 14.4.7 (Microsoft, USA) and were analysed using Stata 13.0, StataCorp

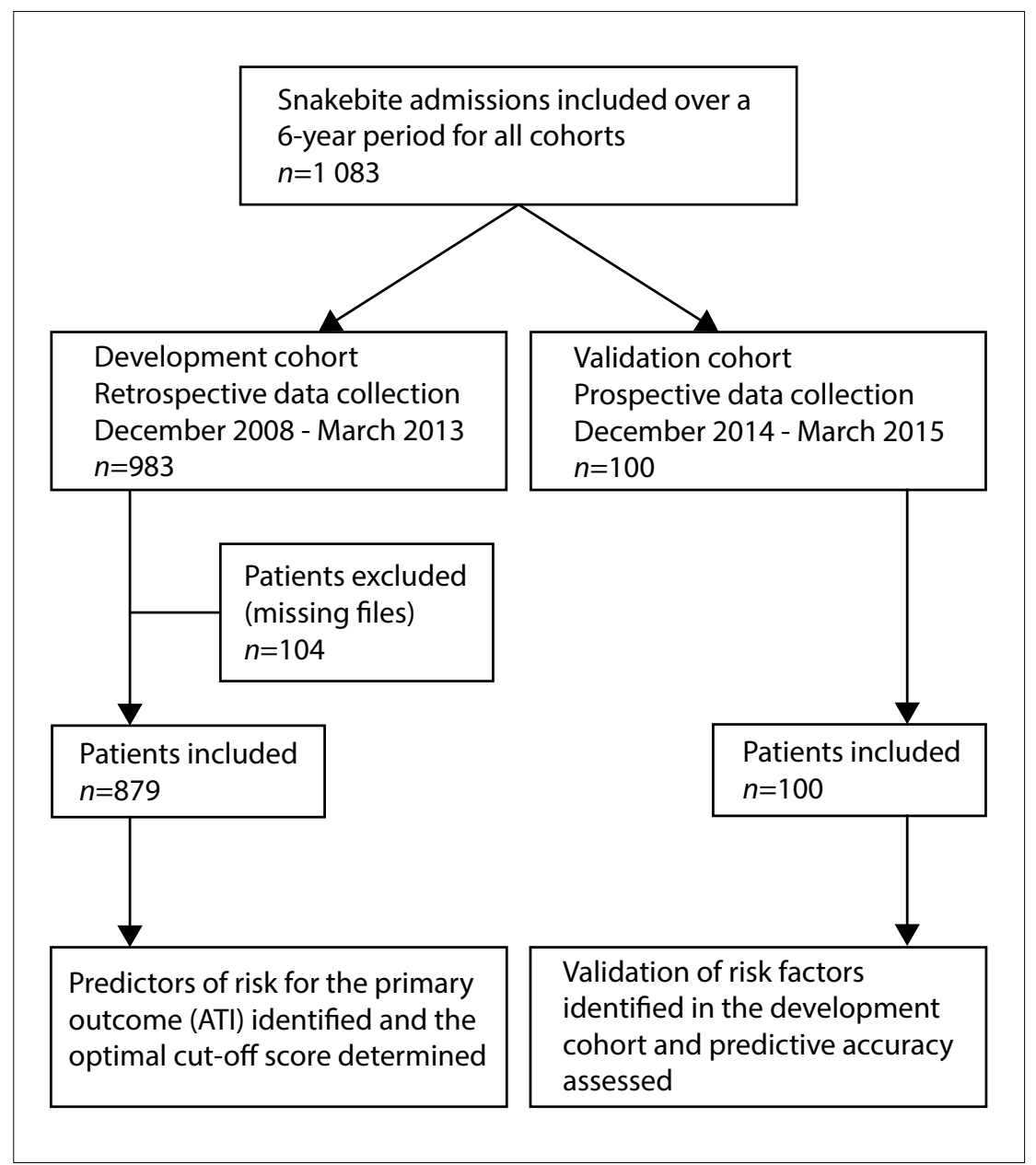

Fig. 1. Patient selection for both development and validation cohorts. 
LP and statistical package 3.0.3 (StataCorp, USA). Associations between ATI and categorical predictors were assessed using a Pearson $\chi^{2}$ or Fisher's exact test. Differences in means of continuous variables were tested using a Student's $t$-test. For non-parametric data the Wilcoxon rank-sum test was used. A $p$-value of $<0.05$ was considered significant. Factors predictive of ATI were identified using bivariate and multivariate analysis and cut-off points determined by ROC curve analysis.

The study was approved by the Biomedical Research Ethics Committee of the University of KwaZulu-Natal (ref. no.: BE034/14).

\section{Results}

We recorded 983 admissions for snakebite over the period December 2008 to December 2013. Of these, 104 were excluded as the clinical records were incomplete; 879 patients were therefore entered into the development cohort. Prospective data for 100 admissions over the period from 1 December 2014 to 31 March 2015 (the snakebite high-incidence season) were used for the validation cohort (the round number of 100 is coincidental). Demographic and laboratory data for both cohorts are presented in Table 1. Of note were significant differences in age, white cell count (WCC) and INR between the two cohorts.

\section{Development cohort}

Of 879 snakebite admissions, 146 reached the primary endpoint of an ATI. Antivenom alone was administered to 64 patients, 49 of 879 patients received a surgical intervention without antivenom and 33 patients received both. Table 2 shows the ATI risk predictors derived from multivariate analysis. Individually no parameter was strongly predictive for ATI. We therefore combined them using a scoring system in which a positive criterion attracted a score of 1. Further testing revealed that weighting the individual criterion according to the number (\%) of cases or the odds ratios (ORs) and using the coefficients derived from the logistic regression did not provide a significantly more accurate prediction than the use of the crude score of 1 each. Therefore, in order to promote ease of use, we settled on a standard score of 1 per positive criterion, to produce a severity score which we named the Zululand Snakebite Score (ZSS) on a six-point scale (Table 3). ROC curve analysis (Fig. 2A) on the minimum number of ATI parameters, returned a value of 4 as the optimal cut-off number for predicting an ATI (area under the curve (AUC) $0.804 ; 95 \%$ confidence interval (CI) $0.758-0.84$ ).

\section{Validation cohort}

Of 100 patients, 40 reached the primary outcome of an ATI. Antivenom alone was administered to 19 patients, 4 patients underwent a surgical intervention without antivenom and 17 patients received both. Within the validation cohort 37 of 100 patients had one or more risk parameters. Applying the ZSS to this cohort, ROC curve analysis (Fig. 2B) confirmed a minimum cut-off score of 4 as the optimal value for predicting an ATI (AUC 0.807; 95\% CI 0.728 0.886). The accuracy of the ZSS in predicting progression to an ATI is reported in Table 4 .

\section{Table 1. Demographic and laboratory data}

\begin{tabular}{|c|c|c|c|}
\hline Characteristic & $\begin{array}{l}\text { Development } \\
\text { cohort }\end{array}$ & $\begin{array}{l}\text { Validation } \\
\text { cohort }\end{array}$ & $p$-value \\
\hline Age (years), mean (SD) & $21.7(15.0)$ & $15.7(13.6)$ & $<0.001$ \\
\hline Children ( $<12$ years), $n(\%)$ & $222(25.3)$ & $57(57.0)$ & $<0.001$ \\
\hline \multicolumn{4}{|l|}{ Sex } \\
\hline Female & $431(49.1)$ & $42(42.0)$ & 0.179 \\
\hline Male & $447(50.9)$ & $58(58.0)$ & \\
\hline Elapsed time between bite and admission (hours), mean (SD) & $9.5(17.4)$ & $10.4(9.6)$ & 0.614 \\
\hline \multicolumn{4}{|l|}{ Elapsed time between bite and admission, $n(\%)$} \\
\hline$<6$ hours & $438(59.0)$ & $47(47.0)$ & 0.023 \\
\hline$\geq 6$ hours & $305(41.0)$ & $53(53.0)$ & \\
\hline Urea $(\mathrm{mmol} / \mathrm{L})$, mean $(\mathrm{IQR})$ & $3.4(2.6-4.2)$ & $3.7(2.8-4.3)$ & 0.216 \\
\hline Creatinine $(\mathrm{mmol} / \mathrm{L})$, mean $(\mathrm{IQR})$ & $63(47-78)$ & $57(36-88)$ & 0.605 \\
\hline WCC $\left(\times 10^{\circ} / \mathrm{L}\right)$, mean $(\mathrm{IQR})$ & $9(6.8-12.0)$ & $12.6(9.0-17.0)$ & $<0.001$ \\
\hline Platelets $\left(\times 10^{9} / \mathrm{L}\right)$, mean $(\mathrm{IQR})$ & $248.5(196-320)$ & $271(204-368)$ & 0.113 \\
\hline INR, mean (IQR) & $1.08(1.01-1.18)$ & $1.20(1.10-1.40)$ & $<0.001$ \\
\hline \multicolumn{4}{|l|}{ Estimation of severity by admitting doctor } \\
\hline Severe & $146(16.6)$ & $26(26.0)$ & 0.019 \\
\hline Not severe & $733(83.4)$ & $74(74.0)$ & \\
\hline
\end{tabular}

Table 2. ATI risk predictors derived from multivariate analysis

\begin{tabular}{|c|c|c|c|c|}
\hline $\begin{array}{l}\text { Calculated ATI risk predictor } \\
\text { and optimal value }\end{array}$ & AUC & OR $(95 \% \mathrm{CI})$ & $p$-value & $\begin{array}{l}\text { ATI cases in which criterion is } \\
\text { positive (\%) }\end{array}$ \\
\hline Age $<14$ yr & 0.657 & $2.13(1.27-3.57)$ & 0.004 & 57 \\
\hline Duration to admission $>7 \mathrm{~h}$ & 0.718 & $4.63(2.71-7.89)$ & $<0.001$ & 53 \\
\hline WCC $>10 \times 10^{9} / \mathrm{L}$ & 0.690 & $3.15(1.88-5.26)$ & 0.023 & 69 \\
\hline INR $>1.2$ & 0.758 & $2.25(1.12-4.53)$ & $<0.001$ & 17 \\
\hline Platelets $<92 \times 10^{9} / \mathrm{L}$ & 0.508 & $2.35(1.01-5.49)$ & 0.048 & 11 \\
\hline Haemoglobin $<7.1 \mathrm{~g} / \mathrm{dL}$ & 0.685 & $5.68(2.14-15.00)$ & $<0.001$ & 3 \\
\hline
\end{tabular}




\begin{tabular}{ll} 
Table 3. Six-point scale Zululand Snakebite Score \\
\hline ATI risk predictors & Allocated score \\
\hline Children $<14$ yr & 1 \\
Duration $>7 \mathrm{~h}$ & 1 \\
$\mathrm{WCC}>10 \times 10^{9} / \mathrm{L}$ & 1 \\
$\mathrm{INR}>1.2$ & 1 \\
Platelets $<92 \times 10^{9} / \mathrm{L}$ & 1 \\
Haemoglobin $<7.4 \mathrm{~g} / \mathrm{dL}$ & 1
\end{tabular}

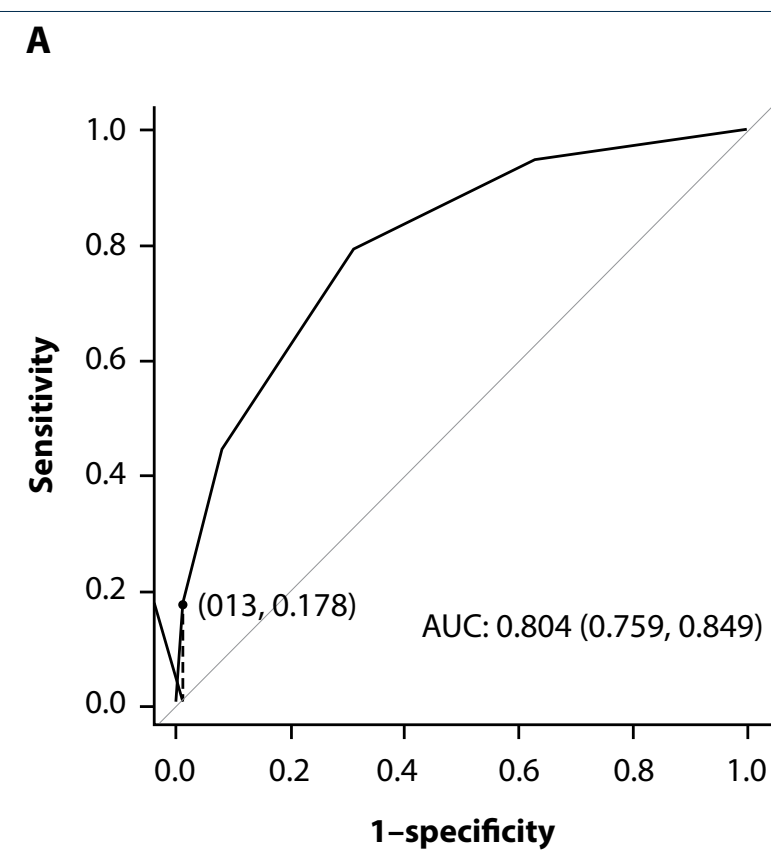

B

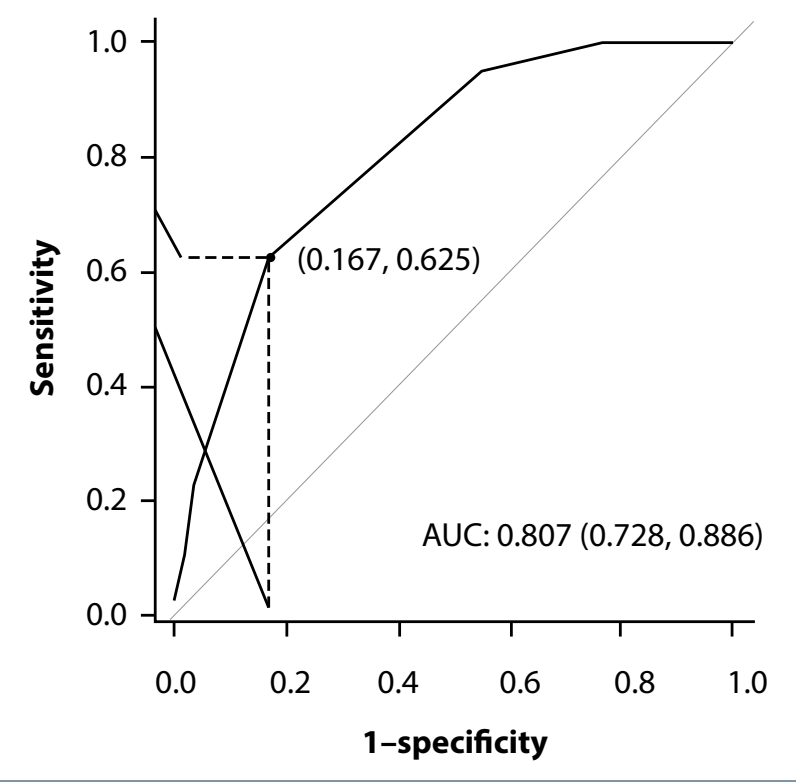

Fig. 2. ROC curve analysis of optimal breakpoint for a crude score of $>4$ as a predictor for ATI. (A) Development cohort ( $\mathrm{N}=879)$. (B) Validation cohort $(\mathrm{N}=100)$.
Table 4. The accuracy of the ZSS in predicting progression to an ATI

\begin{tabular}{lll}
\hline & $\begin{array}{l}\text { Development cohort, \% } \\
\text { (95\% CI) }\end{array}$ & $\begin{array}{l}\text { Validation cohort, \% } \\
(\mathbf{9 5 \%} \text { CI) }\end{array}$ \\
\hline Sensitivity & $17.8(10.9-26.7)$ & $22.5(10.1-38.5)$ \\
Specificity & $98.7(97.3-99.5)$ & $96.6(88.5-99.6)$ \\
PPV & $75.0(57.8-83.4)$ & $81.8(54.4-91.0)$ \\
NPV & $84.9(76.1-93.9)$ & $65.2(44.0-94.0)$
\end{tabular}

\section{Discussion}

Snakebite patients that are obviously severe cases, such as those who present in systemic shock, have active bleeding, manifest neurotoxic muscle weakness or have gross cytotoxic swelling, are easily identified by the admitting doctor, and the decision to prescribe antivenom early or plan for surgery is clinically intuitive. However, it is within the mild to moderate envenomation group that a portion of patients will progress to a severe clinical outcome requiring an ATI. The decision to prescribe antivenom appropriately in these patients is difficult. The risk of anaphylaxis using the South African Institute of Medical Research (SAIMR) polyvalent antivenom in our region is significant; studies report rates of between $25 \%$ and $73 \%$. $^{[1,18-20]}$ Bearing this in mind, doctors must weigh the benefits of antivenom against the risks of anaphylaxis.

Ours is the first attempt to objectively grade snakebites in SA. Our scoring system aims to predict which patients will require more aggressive treatment. The ATI group is intuitively those patients who have had a more severe clinical course, and ATI could possibly act as a surrogate marker for severity. The difficulty in grading the severity of the patient by judging the swelling post envenomation is evident when, in our experience, there is no consistency among doctors who treat snakebites. In our validation cohort 14 of 40 patients who received an ATI were initially assessed as not severe. This supports the view that there is a group of patients that are clinically assessed as non-severe yet progress over time to a more severe course and require an ATI such as antivenom or some form of surgery.

Identifying simple demographic parameters, such as age $<14$ years, delay to admission and noting abnormal admission blood results such as the INR, haemoglobin, WCC and platelets, the doctor can rapidly score a patient and predict whether they will require ATI. In Fig. 3 we propose a new guideline that incorporates both the ZSS and a clinical assessment that is dynamic. ${ }^{[14]}$ The ZSS component will support the doctor's initial clinical assessment in those cases where classifying patients is challenging. Clinicians who score snakebite patients as 4 or more can be reasonably confident that the patient will suffer a more severe clinical course, and will most likely require ATI. If a patient has a positive score, there is an $82 \%$ probability that they will progress to an ATI, based on the positive predictive value (PPV) of the validation cohort. This will alert the doctor to the patient's potential severity and support the decision to prescribe early antivenom. In the case of cytotoxic envenomation, the damage done to tissues can be profound and is not reversible. Thus, there is less damage done to the subcutaneous tissues when a decision to administer antivenom is made early. In many cases this may reduce or prevent surgery of the damaged limb. These results support the ZSS as a 'rule-in' tool for patients who require an ATI.

The lower negative predictive value (NPV) of the ZSS cannot safely rule out those patients with a score of $<4$ as likely to have a non-severe uneventful course. This is a limitation that should be borne in mind 


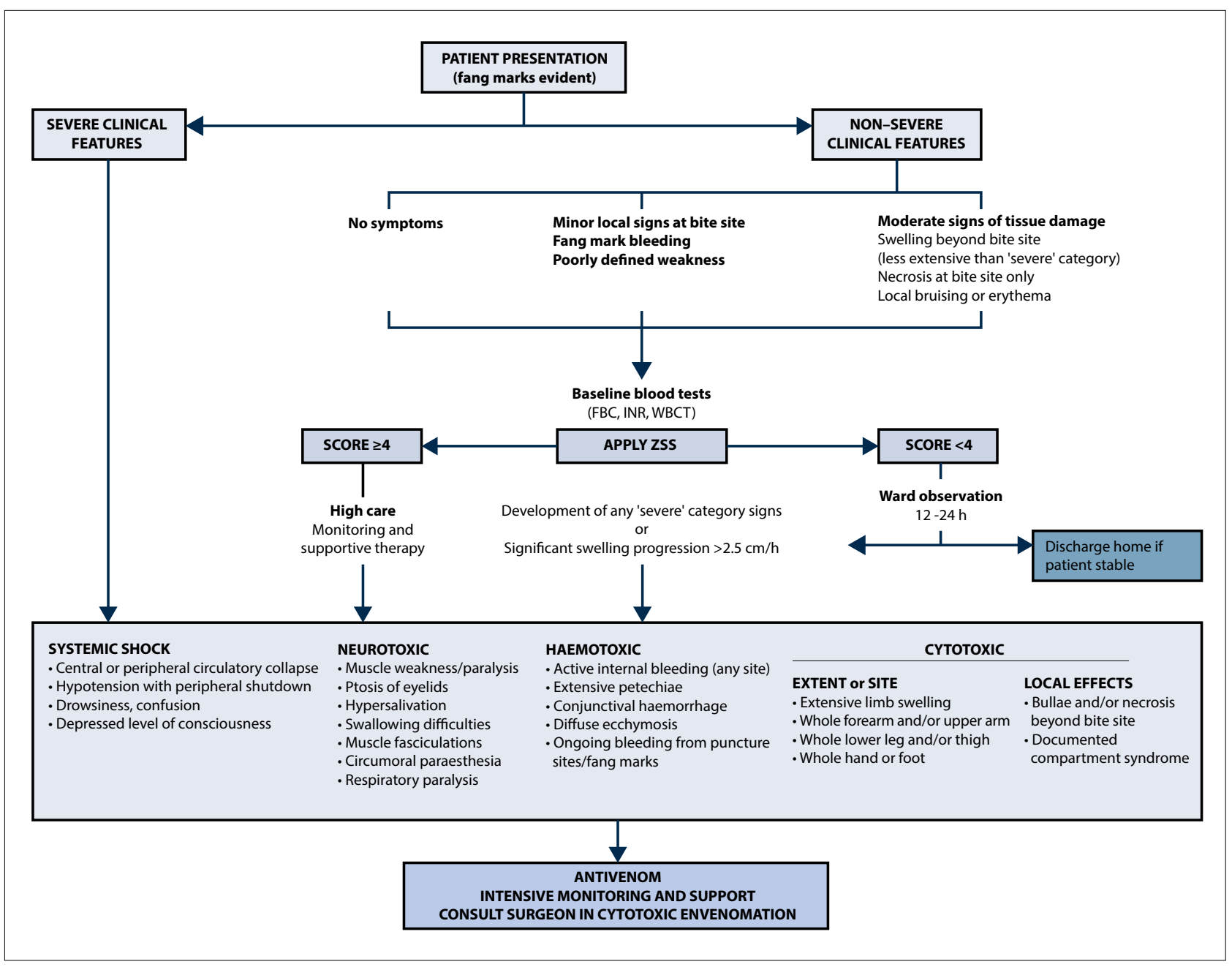

Fig. 3. Proposed guideline for managing snakebites in KwaZulu-Natal. (WBCT = whole blood clotting time; no clotting after 20 min = haemotoxicity (severe category).)

when using the ZSS, and a period of observation is advocated in these patients. Although the patient with a score below 4 has a $65 \%$ probability of not progressing to an ATI, $35 \%$ will actually do so. It is essential that clinicians understand that patients who have a low score should undergo a period of at least 24 hours of observation and monitoring before being discharged.

The risk predictors we identified for ATI, and indirectly severity, are in keeping with reports from other countries. Although the snake species from Asia and the Americas differ from the SA species, there do appear to be some consistent parameters that allude to severity. Age, coagulation abnormalities, low haemoglobin, leukocytosis, thrombocytopenia and delays to treatment are all correlated with adverse outcomes in the Asian and North American viper species. ${ }^{[8,11,12,21,22]}$ It is notable that acute kidney injury is a significant factor in Indian snakebites, while we found no correlation with severity and in our setting acute kidney injury is seldom reported. ${ }^{[16,18,19]}$ One study from southern India reported acute kidney injury in $28 \%$ of snakebite patients, with a significant association with mortality. ${ }^{[23]}$

\section{Study limitations}

We acknowledge the difficulty of clearly defining true severity and the influence that clinical judgement and experience have in influencing a doctor's decision to proceed to an ATI. We believe that the species of snake inflicting the bite may in itself be a potent predictor of the likelihood of a severe outcome. Identification of the species by detecting specific venom antigens might substantially improve prognostication, but these tests are currently not available in SA.

Our sample did not include patients who were treated in other hospitals in the region or patients who were exclusively treated by traditional healers in our local area. We acknowledge that the severity score will have to be validated for use in other areas of southern Africa with a different spectrum of snake species, and may require modification. Until our severity score has been validated (or modified) by other investigators in different settings across SA, we propose to keep its name as the Zululand Severity Score (ZSS); a true SA Severity Score may follow.

\section{Conclusions}

Current guidelines such as those proposed by Blaylock and the Federation of Infectious Diseases of South Africa (FIDSSA) essentially leave the classification of what constitutes a severe snakebite up to the clinician's clinical judgement. ${ }^{[7,24]}$ This research attempts to objectively quantify severe snakebite presentations utilising tested parameters in a scoring system. We hope that such a scoring system will assist doctors who manage snakebite envenomation. 
1. Müller GJ, Modler H, Wium CA, Veale DJH, Marks CJ. Snake bite in southern Africa: diagnosis and management. CME 2012;30:362-382.

2. Bates MF, Branch W, Bauer A, et al. Atlas and Red List of the Reptiles of South Africa, Lesotho and Swaziland. Pretoria: South African National Biodiversity Institute; 2014.

3. Hayes WK, Herbert SS, Rehling GC, Gennaro JF. Factors that influence venom expenditure in viperids and other snake species during predatory and defensive contexts. In: Schuett GW, Hoggren M, Douglas ME, eds. Biology of the Vipers. Eagle Mountain, Utah: Eagle Mountain Publications, 2002:207-233

4. Alirol E, Sharma SK, Bawaskar HS, Kuch U, Chappuis F. Snake bite in South Asia: A review. PLoS Negl Trop Dis 2010;4:e603. http://dx.doi.org/10.1371/journal.pntd.0000603

5. Gold BS, Dart RC, Barish RA. Bites of venomous snakes. N Engl J Med 2002;347:347-356. http://dx.doi. 5. Gold BS, Dart RC, Barish RA.
org/10.1056/NEJMra013477

org/10.1056/NEJMra013477
6. Juckett G, Hancox JG. Venomous snakebites in the United States: Management review and update. Am 6. Juckett G, Hancox JG. Venomous sn
Fam Physician 2002;65:1367-1378.

Fam Physician 2002;65:1367-1378.
Blaylock R. The identification and syndromic management of snakebite in South Africa. S Afr Fam Practice 2005;47:48-53. http://dx.doi.org/10.1080/20786204.2005.10873288

8. Dadpour B, Shafahi A, Monzavi SM, et al. Snakebite prognostic factors: Leading factors of weak therapeutic response following snakebite envenomation. Asia Pacific J Med Toxicol 2012;1(1):27 33 .

9. Ertem K, Esenkaya I, Kaygusuz MA, Turan C. Our clinical experience in the treatment of snakebites. Acta Orthop Traumatol Turc 2005;39:54-58.

10. Monzavi SM, Dadpour B, Afshari R. Snakebite management in Iran: Devising a protocol. J Res Med Sci 2014;19:153.

11. Moriarity RS, Dryer S, Repogle W, Summers RL. The role for coagulation markers in mild snakebite envenomations. West J Emerg Med 2012;13(1):68-74. http://dx.doi.org/10.5811/westjem.2011.6.6729

12. Dart RC, Hurlbut KM, Garcia R, Boren J. Validation of a severity score for the assessment of crotalid Dart RC, Hurlbut KM, Garcia R, Boren J. Validation of a severity score for the assessment of
snakebite. Ann Emerg Med 1996;27:321-326. http://dx.doi.org/10.1016/S0196-0644(96)70267-6

13. Scharman EJ, Noffsinger VD. Copperhead snakebites: Clinical severity of local effects. Ann Emerg Med 2001;38(1):55-61. http://dx.doi.org/10.1067/mem.2001.116148
14. Lavonas EJ, Ruha AM, Banner W, et al. Unified treatment algorithm for the management of crotaline snakebite in the United States: Results of an evidence-informed consensus workshop. BMC Emerg Med 2011:11(1):2. http://dx.doi.org/10.1186/1471-227X-11-2

15. Wood D, Sartorius B, Hift R. Snakebite in north-eastern South Africa: Clinical characteristics and risks for severity. S Afr Fam Practice 2016;58(2):62-67. http://dx.doi.org/10.1080/20786190.2015.1120934

16. Blaylock R. Epidemiology of snakebite in Eshowe, KwaZulu-Natal South Africa. Toxicon 2004:43:159. http://dx.doi.org/10.1016/j.toxicon.2003.11.019

17. Blaylock RS. Retrospective analysis of snakebite at a rural hospital in Zululand. S Afr Med J 1995;85:286 18. Coetzer PW, Tilbury CR. The epidemiology of snakebite in northern Natal. S Afr Med J 1982;62:206212.

19. Wood D, Webb C, DeMeyer J. Severe snakebites in northern KwaZulu-Natal: treatment modalities and outcomes. S Afr Med J 2009;99:814-818

20. Moran NF, Newman WJ, Theakston RDG, Warrell DA, Wilkinson D. High incidence of early anaphylactoid reaction to SAIMR polyvalent snake antivenom. Trans R Soc Trop Med Hyg 1998;92:69-70. http://dx.doi. org/10.1016/S0035-9203(98)90959-2

21. Sankar J, Nabeel R, Sankar MJ, Priyambada L, Mahadevan S. Factors affecting outcome in children with snake envenomation: a prospective observational study. Arch Dis Child 2013;98:596-601. http:// dx.doi.org/10.1136/archdischild-2012-303025

22. Suchithra N, Pappachan I, Sujathan P. Snakebite envenoming in Kerala, South India: Clinical profile and factors involved in adverse outcomes. Emerg Med 2008;25:200-204. http://dx.doi.org/10.1136/ and factors involved in adverse outcomes. Emerg Med 2008;25:200-204. http://dx.doi.org/10.1136/ emj.2007.05113

23. David S, Matathia S, Christopher S. Mortality predictors of snake bite envenomation in southern India - a ten-year retrospective audit of 533 patients. J Med Toxicol 2012;8:118-123. http://dx.doi.org/10.1007/ 13181-011-0204-0

24. Cohen P. 2010. Management of snakebite. http://www.fidssa.co.za/Content/Documents/Mangement $\% 20$ of\%20Snakebite.pdf (accessed 1 December 2016).

Accepted 26 September 2016 